\title{
Contamination de Pont-l'Evêque par un Penicillium provenant des boîtes et cageots servant à leur emballage
}

\author{
par \\ M. DESFLEURS \\ Ingénieur chimiste I.C.L., Docteur ès sciences $3^{\mathrm{e}}$ cycle. \\ Institut du Lait, des Viandes et de la Nutrition \\ de l'Université de Caen
}

En Juin 1974, les fromages Pont-l'Evêque d'une fromagerie de l'Orne furent envahis par une moisissure bleu-vert. Les responsables de la fabrication, en cherchant les causes de cette infection s'aperçurent que le bois d'un certain nombre de boîtes et cageots servant à l'emballage de ces fromages présentaient des taches de moisissures. Sur un milieu nutritif, ils déposèrent quelques fragments du bois contaminé et obtinrent ainsi un développement mycélien. La culture nous fût soumise accompagnée d'un prélèvement direct des fromages de Pont-l'Evêque.

Après nouveaux isolements et cultures, nous pûmes constater qu'il s'agissait d'un Penicillium identique dans tous les cas.

A la même époque, le chef de fabrication d'une fromagerie de la Mayenne, nous signala que ses Pont-l'Evêques souffraient d'un accident analogue. Cette fromagerie produisant aussi des Camemberts, ceux-ci se trouvèrent rapidement contaminés, plus sur la face du ressalage que sur l'autre, d'ailleurs.

Comme prévisible, nous trouvâmes encore la même espèce de Penicillium responsable des envahissements des deux fromages, mais fait plus curieux, nous avions affaire à l'espèce déjà trouvée dans l'Orne.

Enfin, le prélèvement d'une moisissure bleu-vert que nous fîmes sur un Pont-l'Evêque fabriqué dans la Manche, nous livra, là encore, le même Penicillium. 


\section{A. - CARACTERES MICROSCOPIQUES ET CULTURAUX}

$1^{\circ}$ Sur milieu de Czapek, les colonies de ce champignon à $20^{\circ} \mathrm{C}$ atteignent un diamètre de 3 à $4 \mathrm{~cm}$ en une douzaine de jours. Elles s'accroissent en présentant, d'abord, un bord blanc de $1 \mathrm{~mm}$ de large, suivi d'une marge vert-bleuté pâle d'environ $2 \mathrm{~mm}$; puis la couleur passe au marron jaunâtre clair jusqu'au centre surélevé qui reste verdâtre. Elles sont très sporulées, granuleuses, zonées, avec un exsudat de fines gouttelettes incolores. L'odeur de moisi est forte. Le revers, d'abord blanc, devient crème, puis finit jaunâtre.

Les pinceaux des appareils conidiens sont asymétriques, non divergeants et portent de longues chaînes de conidies, en colonnes parallèles qui s'emmèlent rapidement en masses. Les conidiophores sont habituellement issus d'hyphes traînant sur le substrat. Ils ont, généralement, une hauteur de $280 \mu$ avec un diamètre de $5 \mu$. Leurs parois sont granuleuses. On trouve des conidiophores non branchés, mais, le plus souvent, ils ont une ou deux branches adjointes à la tige principale, d'une longueur de 21 à $32 \mu$, en moyenne $24 \mu$, la largeur étant de $3,5 \mu$.

Ces branches portent des verticilles de métules de 12 à $14 \mu$ de long, mais parfois de 17 à $24 \mu$, par $3,5 \mu$. Elles se terminent par un verticille de 4 à 5 phialides de 7 à $12 \mu$ de long (en moyenne 10,5 $\mu$ ) par $3,5 \mu$ de large.

Les conidies sont globuleuses, d'un diamètre de 3 à $3,5 \mu$. Leur paroi est apparemment lisse. Cependant si, après les avoir fixées, d'abord, par le formol, puis ensuite par les vapeurs d'acide osmique, ainsi que nous l'avons recommandé [2] pour les empêcher de gonfler sous l'effet du vide, on les regarde au microscope électronique, elles apparaissent très légèrement bosselées. La sorte de gaine qui les réunit en chaînes, tout en les séparant, et que l'on nous permettra d'appeler " disjoncteur », est large.

$2^{\circ}$ Les colonies sur milieu glucosé à l'extrait de levure, ne diffèrent pas sensiblement des précédentes, si ce n'est qu'elles restent vert bleuâtre et ne deviennent gris marron, parfois légèrement verdâtre, que très tardivement, pendant que le revers passe du jaunâtre à l'ocre, et même, au marron un peu rougeâtre.

$3^{\circ}$ Sur milieu au malt gélosé, les colonies ne sont, d'abord, constituées que par du mycélium incolore partant en rayons du point d'ensemencement. Puis, ce centre devient vert bleuté, légèrement surélevé, tandis que les colonies poussent très rases, peu sporulées, présentant des granulations vert bleuâtre peu foncé. Elles atteignent un diamètre de $4 \mathrm{~cm}$ en une douzaine de jours, à $20^{\circ} \mathrm{C}$. L'odeur, rappelant celle des champignons, est forte. Le revers, d'abord inco- 
lore, s'ocre en vieillissant. Les conidiophores portent de très longues chaînes de conidies en colonnes parallèles ou qui s'emmèlent.

\section{B. - DETERMINATION}

Ce Penicillium est asymétrique - Ses colonies sont légèrement granuleuses sur tous les milieux - Il se range donc parmi les Asymmetrica fasciculata.

Etant donné sa couleur sur milieu de Czapek, on pense de suite au $P$. ochraceum, ce qui serait exact si la teinte marron clair persistait sur les autres milieux, mais elle y est vert bleuâtre sur ceux-ci. Raper et Thom écrivent [7] : «Bien que le $P$. ochraceum représente une série basée primitivement sur la couleur non verte de ses conidies, l'attention doit être attirée sur la tendance de cette espèce à se confondre avec la série du $P$. viridicatum. La relation probable ne doit pas être négligée ». Et encore : «Il y a une évidence considérable qui appuie la conviction que les séries du $P$. psittacinum, $P$. ochraceum et $P$. viridicatum, appartiennent toutes à un groupe simple, mais extrêmement variable, dans lequel les changements de couleur du jaune vert au brun olive, représentent une caractéristique fondamentale ". Enfin, parlant de la série du $P$. viridicatum qui présente habituellement une couleur jaune vert, ils disent : " La teinte bleuâtre est généralement transitoire. Les colonies deviennent souvent brun clair en vieillissant ".

C'est le cas du Penicillium que nous étudions. Nous le rangerons donc dans le groupe du P. viridicatum Westling, qui comporte, aussi, certaines souches se rapprochant du $P$. expansum.

Les différences entre les caractéristiques du $P$. viridicatum type et celles de ce champignon sont minimes, sauf la couleur. Ses pinceaux ordinairement sont peut-être moins larges. Pourtant les différents articles qui les composent ont les mêmes dimensions, chez l'un et l'autre. Les photographies au microscope électronique de ses conidies, identiques à celles de Sakaguchi et Abe [8], les montrent très légèrement raboteuses, avec un " disjoncteur » large, ce qui est, à notre sens, caractéristique.

\section{C. - BIOLOGIE DU CHAMPIGNON ORIGINE ET PREVENTION DE L'ACCIDENT}

Le $P$. viridicatum est une moisissure du sol qui participe activement à la décomposition des résidus organiques. Rien de plus naturel que de le trouver sur du bois. Nous avions signalé qu'il pouvait croître sur les fromages [2]. Il pousse aussi sur les fruits, les grains 
trop humides, blés, maïs ; Marchionatto [4, 5] le signale comme responsable sur le maïs, en Argentine, de l'empoisonnement de porcs et de chevaux. Il est nocif par l'élaboration d'un antibiotique, la viridicatine $\mathrm{C}_{15} \mathrm{H}_{11} \mathrm{O}_{2} \mathrm{~N}$, selon Moreau [6].

Chez la souris, Carlton et al. [1] disent qu'il réduit les gains de poids par rapport aux témoins, augmente la mortalité et provoque des lésions hépatiques microscopiques et macroscopiques.

On ne peut rien en déduire quant au $P$. viridicatum que nous avons isolé - Au sein d'une même espèce, certaines souches sont toxiques, alors que d'autres sont inoffensives - De plus, ici, nous avons affaire, non au type lui-même, mais à une de ses variantes.

Si les caractéristiques particulières de ce Penicillium nous ont rendu malaisé son classement, par contre elles nous permettent d'affirmer avec certitude l'identité des cinq isolements effectués. Mais, la simultanité de l'infection dans ces trois fromageries éloignées les unes des autres, ne peut s'expliquer que parce qu'elles ont toutes le même fournisseur de boîtes et que ce $P$. viridicatum particulier en provient. Nous y avons retrouvé les spores en abondance et même des colonies installées sur le bois. Nous avons vu aussi qu'il « poudre " beaucoup, c'est-à-dire que la manipulation du support contaminé - boîtes, fromages - provoque un nuage de conidies que l'air se charge de disséminer rapidement.

La désinfection des locaux par le formol, les traitements appropriés de surface, qui peuvent être appliqués sur le Pont-l'Evêque, amenèrent la disparition de cet accident.

Avec le professeur Jacquet nous avions déjà attiré l'attention sur le rôle possible des emballages dans la contamination fongique des fromages à pâte molle [3]. En voici un autre exemple qu'il nous a paru intéressant de rapporter.

Pour éviter cette infection, il faudrait exiger que le fabricant livre des boîtes dont le bois n'est pas moisi. Même s'il est étuvé, ce qui n'est pas le cas pour les cageots, il faut qu'ensuite, il soit conservé à l'abri et dans des conditions telles qu'elles ne favorisent pas le développement de ces champignons microscopiques. Ceux-ci sont d'espèces variées car, outre les Penicilliums, nous y avons trouvé des Mucor, Rhizopus, Scopulariopsis fusca, Monilia sitophila, Stemphylium lanuginosum. On nous a d'ailleurs signalé, en décembre 1974, qu'un autre arrivage de boîtes a, de nouveau, provoqué un début d'infection.

Enfin, sur la prévention, nous reprendrons les conseils que nous avions déjà donnés dans l'article précité [3] : stocker les papiers et les boîtes dans des locaux spéciaux, très secs, parfaitement distincts de ceux de fabrication. Ces magasins ne devraient communiquer qu'avec la salle d'emballage et comme celle-ci, être désinfectés régulièrement et périodiquement par le formol. 


\section{Ré s u mé}

Dans trois fromageries différentes, l'auteur a trouvé sur les Pont-l'Evêques qu'elles fabriquent, le $P$. viridicatum provenant des emballages en bois. Il en donne la description et précise les moyens de s'en préserver.

\section{S u $\mathrm{m} \mathbf{m}$ a $\mathbf{r}$ y}

In three different cheese factories, the author found on the Pont-l'Evêque cheeses, the $P$. viridicatum which came from the wooden packings. He gives its description and how to be protected from it.

Reçu en avril 1975 pour publication.

\section{Bibliographie}

[1] Carlton (W. W.) et al. (1968). - Investigations of the toxic effects in mice of certain species of Penicillium. Toxic. appl. pharm., 13, 372-387.

[2] Desfleurs (M.) (1966). - Contribution à la connaissance du genre Penicillium. Application à la fabrication des fromages à pâte molle et notamment du Camembert. Thèse de Doctorat de $3^{\mathrm{e}}$ cycle Fac. Sc. de Caen.

[3] Jacquet (J.) et Desfleurs (M.) (1966). - Scopulariopsis brevicaulis Bainier ou mieux Scopulariopsis fusca Zach, agent de taches superficielles brun violet des fromages à pâte molle. Le Lait, 46, 241-253.

[4] Marchionatto (J.) (1941). - El moho del maiz. J. Agron., Buenos Aires.

[5] Marchionatto (J.) (1942). - El verdin del maiz. Rev. Fac. Agron., Buenos Aires, 9, 159-169.

[6] Moreau (Cl.) (1973). - Moisissures toxiques dans l'alimentation. Un vol., Masson éd., Paris, $2^{\mathrm{e}}$ édition.

[7] Raper (K.) and Thom (C.) (1949). - A manual of the Penicillia. Baillière, Tindall et Cox éd. Londres.

[8] SaKaguchi (K. I.) and ABE (S.) (1957). - Atlas of microorganismes the Penicillia. Kanehara Shuppan éd., Tokyo. 